\title{
Tumor-secreted Pros1 inhibits macrophage M1 polarization to reduce antitumor immune response
}

\author{
Eric Ubil, ${ }^{1}$ Laura Caskey, ${ }^{1}$ Alisha Holtzhausen, ${ }^{1}$ Debra Hunter, ${ }^{1}$ Charlotte Story, ${ }^{1}$ and H. Shelton Earp ${ }^{1,2}$ \\ 'UNC Lineberger Comprehensive Cancer Center and 'Departments of Medicine and Pharmacology, University of North Carolina at Chapel Hill (UNC), Chapel Hill, North Carolina, USA.
}

\begin{abstract}
Tyro3, AxI, Mer (TAM) receptor tyrosine kinases reduce inflammatory, innate immune responses. We demonstrate that tumor-secreted protein S (Pros1), a Mer/Tyro3 ligand, decreased macrophage M1 cytokine expression in vitro and in vivo. In contrast, tumor cells with CRISPR-based deletion of Pros1 failed to inhibit M1 polarization. Tumor cell-associated Pros1 action was abrogated in macrophages from Mer- and Tyro3- but not AxI-KO mice. In addition, several other murine and human tumor cell lines suppressed macrophage M1 cytokine expression induced by IFN- $\gamma$ and LPS. Investigation of the suppressive pathway demonstrated a role for PTP1b complexing with Mer. Substantiating the role of PTP1b, M1 cytokine suppression was also lost in macrophages from PTP1b-KO mice. Mice bearing Pros1-deficient tumors showed increased innate and adaptive immune infiltration, as well as increased median survival. TAM activation can also inhibit TLR-mediated M1 polarization. Treatment with resiquimod, a TLR7/8 agonist, did not improve survival in mice bearing Pros1-secreting tumors but doubled survival for Pros1-deleted tumors. The tumor-derived Pros1 immune suppressive system, like PD-L1, was cytokine responsive, with IFN- $\gamma$ inducing Pros1 transcription and secretion. Inhibition of Pros1/TAM interaction represents a potential novel strategy to block tumor-derived immune suppression.
\end{abstract}

\section{Introduction}

Certain cancer cells subvert the immune response by eliciting an environment that favors immunologic tolerance. This immuneprivileged status is achieved by mimicking existing homeostatic processes that mitigate immune responses, including those that prevent chronic inflammation and autoimmunity. The adaptive immune checkpoint genes programmed cell death ligand 1 (PD-L1) (1) and cytotoxic T lymphocyte-associated protein 4 (CTLA4) (1) are prime examples. Both fundamental and clinical studies are focusing on ways to reverse suppression and boost cancer-specific immunity (2).

Tumor-associated macrophages, which have been linked to increased tumor progression and metastasis, are an emerging target for therapeutic intervention $(2,3)$. Intratumoral macrophages can be derived from existing tissue-resident macrophages or tumor-elicited bone marrow cells that traffic to the tumor and differentiate into macrophages. In early tumorigenesis, resident macrophages could be part of a first response and, in association with other innate immune cells, initiate an inflammatory response that either coordinates an adaptive immune response or in some contexts facilitates progression. In established tumors, either resident macrophages or bone marrow-derived macrophages (BMDMs) tend to be polarized toward an M2, or alternatively activated, phenotype rather than the inflammatory M1 state $(4,5)$. This M2 wound-healing phenotype promotes tumor progression and metastasis by secreting growth and angiogenesis factors, remod-

Conflict of interest: HSE is a cofounder of the UNC startup Meryx, which licenses intellectual property from UNC Chapel Hill on the design of small molecule kinase inhibitors for members of the Tyro3, Axl, and Mer family.

Submitted: September 6, 2017; Accepted: March 13, 2018.

Reference information: J Clin Invest. 2018;128(6):2356-2369.

https://doi.org/10.1172/JCI97354. eling extracellular matrix, and suppressing immune responses (4, $6,7)$. In contrast, M1-activated macrophages reduce tumor progression, inhibiting tumor growth through direct action, such as secretion of ROS, or promotion of the Th1 response $(4,7)$.

Members of the Tyro3/Axl/Mer (TAM) family of receptor tyrosine kinases (RTKs) are key modulators of the innate immune system and are essential for efficient clearance of apoptotic material during normal homeostatic processes (8-11). TAM receptors are activated by ligands: Gas6, protein S (Pros1), Tubby, Tulp-1, or galectin-3. The best-studied TAM ligands, Gas6 and Pros1, form a bridge between exposed phosphatidylserine (PtdSer), externalized on the surface of apoptotic cells, and the macrophage TAM receptors to trigger efferocytosis (11). While ligand complexing with PtdSer is not necessary to activate TAM receptors, it significantly increases the affinity of the ligand for the receptor, enhancing RTK activation (12).

Our previous studies suggest that tumors manipulate Mer activity on innate immune cells, as genetic knockout of Mer increased tumor latency and reduced metastases in syngeneic murine melanoma (B16F10) and breast cancer (PyMT) (13). Bone marrow transplant studies further demonstrated that Merdeficient leukocytes conferred this tumor resistance, increased proinflammatory responses, and reduced expression of antiinflammatory cytokines (13). However, the mechanism(s) by which tumor cells utilize TAM receptors to downregulate the immune response needs further elucidation.

Here we show that tumor cells secrete Pros1 and effectively dampen macrophage M1-associated gene expression through Mer and Tyro3. We address a new mechanism: Pros1 signaling reduces macrophage p38 $\alpha$ activity through an induced complex between Mer, p38a, and protein tyrosine phosphatase 1B (PTP1b). Loss of Mer or pharmacological PTP1b inhibition restored M1 gene 
expression. In vivo studies demonstrated that increased immune infiltration occurs when B16F10 cells bearing CRISPR-based deletion of Pros1 form tumors as compared with intact B16F10 tumors. The heightened immune infiltration to the Pros1-deleted tumor is associated with reduced tumor viability, particularly in animals cotreated with a TLR agonist. Last, we show that in cancer cells from several tissue origins, Pros1 expression (like that of PD-L1) is induced by IFN- $\gamma$, demonstrating that the inflammatory environment puts in motion tumor-derived mechanisms capable of suppressing both innate and adaptive immunity. These results suggest that Pros1 and TAM RTKs could be targeted as innate immune checkpoint functions in cancer.

\section{Results}

Tumor-secreted Pros1 suppresses macrophage M1 polarization. Our group and colleagues previously demonstrated that Mer deficiency in host leukocytes increases resistance to tumor progression and reduces metastases $(13,14)$, suggesting that the tumor microenvironment activates Mer signaling, presumably by a combination of apoptotic cells and exosomes plus an undiscovered source of bridging ligand. To examine in vivo expression of 2 primary ligands during tumor initiation and growth, we implanted luciferasetagged B16F10 melanoma cells subcutaneously, harvested the resultant tumor, and immunostained for Gas6 or Pros1. Tumor cells expressing Gas6 increased over time from $24 \% \pm 5 \%$ one day after implantation to $85 \% \pm 3 \%$ by day 10 . Pros1, however, was expressed by $79 \% \pm 5 \%$ of tumor cells within 24 hours, and levels stayed uniform over the course of 10 days (Figure 1A). Although Mer activation in human macrophages can increase Gas6 RNA levels, a type of feed-forward autocrine ligand upregulation (15), in this in vivo model the TAM ligands were expressed predominantly by tumor cells with $98 \% \pm 2 \%$ of immunostainable Gas 6 and $96 \% \pm 3 \%$ of Pros1 localized to B16F10 cells (Figure 1A).

To better understand tumor ligand/innate immune cell receptor interactions, we developed a Transwell coculture system in which tumor cells and harvested resident peritoneal macrophages were cultured in the same well though separated by a $0.4-\mu \mathrm{m}$-poresize barrier. This enabled study of paracrine signaling through tumor-secreted TAM ligands without physical interaction of the 2 cell types. M1 activation was induced by treating macrophages with IFN- $\gamma$ and LPS (5). Prior to coculture, we determined whether IFN- $\gamma$ or LPS altered B16F10 TAM ligand expression. IFN- $\gamma$, but not LPS, increased Pros 1 expression $5 \pm 0.5$-fold (Figure 1B). The findings were reminiscent of recent reports demonstrating that PD-L1 expression is induced by IFN- $\gamma$ (16-18), suggesting a broader tumor-derived (adaptive and innate) immune-suppressive program initiated in response to IFN $-\gamma$. The increase in mRNA was accompanied by a $38 \%$ increase in Pros 1 secretion to $1.7 \mu \mathrm{g} / \mathrm{ml}$ (22 nM) measured by ELISA in conditioned medium (Figure 1C). This effect was generalizable, with Pros 1 mRNA increasing in 6 of 8 melanoma, lung, breast, and pancreatic cell lines by 1.5- to 4.2-fold in response to IFN- $\gamma$ (Supplemental Figure 1A; supplemental material available online with this article; https://doi. org/10.1172/JCI97354DS1). Conversely, Gas6 transcription was decreased in B16F10 cells after treatment with IFN- $\gamma$ (Figure 1B).

Using IFN- $\gamma$ and LPS as a model for M1 induction, we determined the effect of Gas6 and Pros1 treatment on peritoneal mac- rophage polarization. Treatment of M1-polarized macrophages with $1 \mu \mathrm{g} / \mathrm{ml}$ recombinant human Pros1 (80\% homologous to murine Pros1) for 24 hours suppressed expression of M1-associated genes by as much as $43 \%$ (Figure 1D and Supplemental Figure 2), while treatment with $200 \mathrm{ng} / \mathrm{ml}$ Gas6, which has been previously reported to activate TAM signaling (19), did not significantly alter transcription of M1 genes (Supplemental Figure 3). Pros1 produced different suppressive effects on the mRNA levels of specific cytokines; IL1 and IL6 were most sensitive, CD86 and TNFa were less sensitive, and iNOS was insensitive. The results were similar when instead of being treated with exogenous Pros1, B16F10 cells were cocultured with peritoneal macrophages. Coculture for 24 hours produced as much as a $57 \%$ decrease in IFN- $\gamma$ and LPS-stimulated M1 gene expression (Figure 1D and Supplemental Figure 2). To establish how rapidly Pros1 suppresses M1 gene transcription in our in vitro model, we also measured M1 mRNA induction at 2 and 8 hours in the presence of exogenous Pros1 or during coculture with B16F10. While we did not observe Pros1-mediated M1 suppression after 2 hours, it was observed by 8 hours for IL1 and IL6 (65\% $\pm 6 \%$ and $31 \% \pm 11 \%$, respectively), and by 24 hours suppression was also observed for CD86 and TNFa (Supplemental Figure 4 and Figure 1D). Our results show that while Pros1 does not immediately suppress M1 polarization, within 8 hours a time-dependent process initiates suppression of M1-associated gene expression. This suggests that Pros1 expression and secretion may act as one mechanism to resolve inflammation during normal physiological processes.

In these tissue-derived peritoneal macrophages, M2-associated gene expression was unchanged (Supplemental Figure 5), demonstrating that in this macrophage population, Pros1 suppresses M1 cytokine production without skewing them toward an M2 phenotype.

M1 polarized thioglycollate-induced peritoneal macrophages, which comprise primarily bone marrow-derived cells, showed a similar reduction in M1 gene expression, with as much as $41 \%$ $\pm 6 \%$ or $74 \% \pm 6 \%$ suppression after Pros 1 treatment or coculture with B16F10, respectively (Supplemental Figure 6), with the exception of CD86. Our findings show that regardless of macrophage origin, Pros1 reduces M1-associated gene expression.

Extending our finding to other murine cancers, we cocultured M1-induced macrophages with various tumor cell lines and observed suppression ranging from as much as $70 \%$ (Lewis lung carcinoma) to none at all (PyMT) (Supplemental Figure 1B). To determine whether differences in M1 suppression could be attributed to the amount of tumor secreted Pros1, we cultured tumor cell lines for 24 hours and measured Pros1 concentrations in their respective conditioned medium by ELISA (Supplemental Figure 1C). The immunoassay concentration of Pros1 did not explain the differences in suppression, though the varying extent of M1 gene suppression by different cell lines may be attributed to a number of factors, including the ability of the different cells to appropriately synthesize and fold the vitamin K-dependent domain or mutations affecting Pros1 structure or function.

Further extending our findings to human cancer, we performed our coculture assay using the human macrophage cell line SC and 6 different human tumor cell lines, representing melanoma (G361, HMCB), breast (MCF7, MDA-MB-231), and prostate cancers (LNCap, PC3). Strikingly, melanoma and breast cancer cell lines suppressed IFN- $\gamma /$ LPS stimulated M1 gene expression 

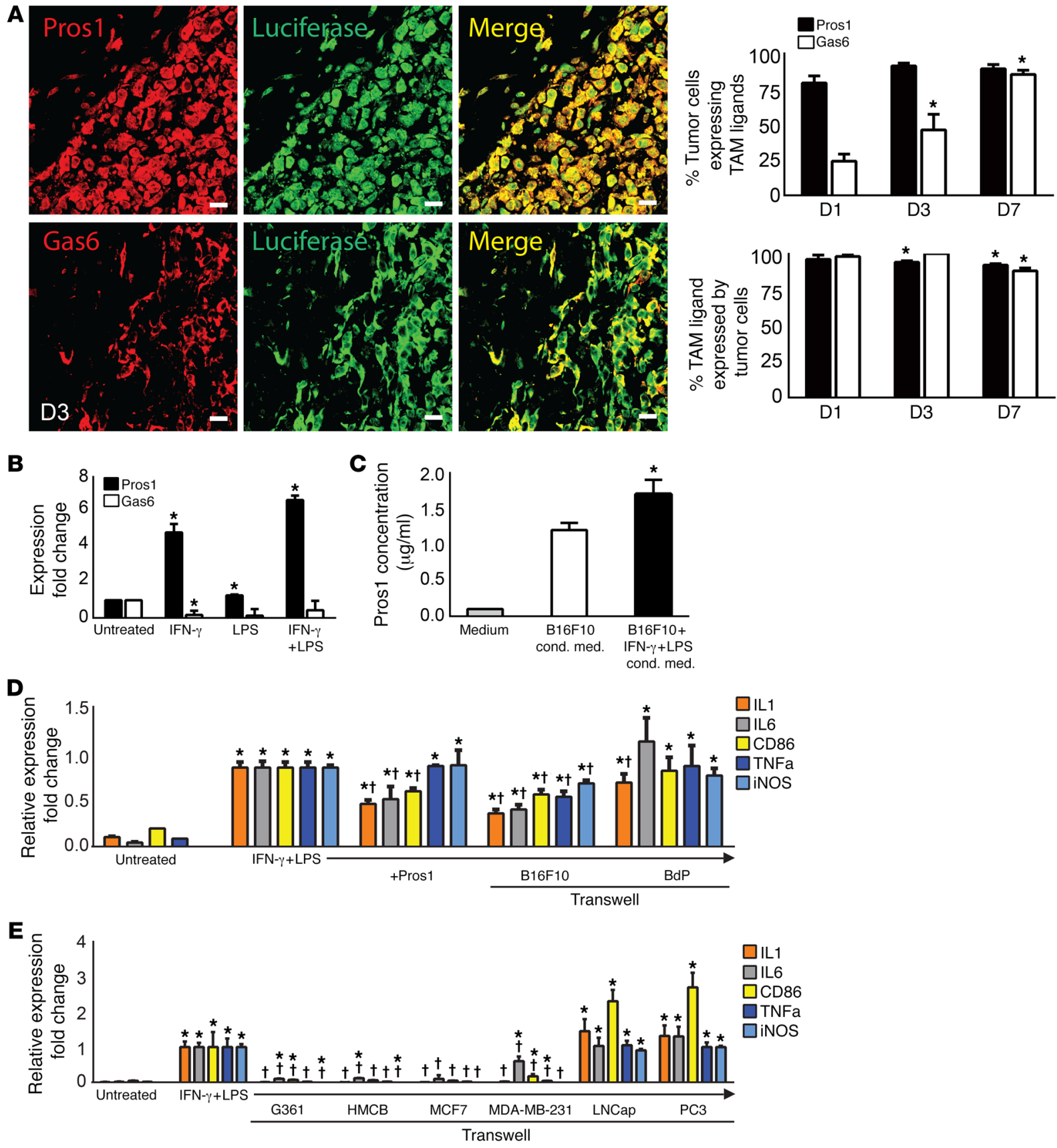

Figure 1. Tumor-secreted Pros1 inhibits macrophage M1 polarization. (A) Immunofluorescence staining and colocalization analysis of dynamic expression of TAM ligands in luciferase-tagged B16F10 tumor cells (day 3 [D3] after implantation, scale bars: $20 \mu \mathrm{m}, n=5,{ }^{*} P<0.05$ relative to day 1 ). (B) B16F10 expression of TAM ligands in immune activating conditions as measured by qRT-PCR. $n=6,{ }^{*} P<0.05$ relative to untreated, 2 independent replicates. (C) ELISA of Pros1 secretion by B16F10 cells. $n=4,{ }^{*} P<0.05$ relative to B16F10 conditioned medium (cond. med.), 2 independent replicates. (D) Suppression of M1-associated gene expression by recombinant human or tumor-secreted Pros 1 measured by qRT-PCR. $n=10,{ }^{*} P<0.05$ relative to untreated, ${ }^{\dagger} P<0.05$ relative to M1-induced, 4 independent replicates. (E) Suppression of M1-associated gene expression in human macrophage cell line (SC) cocultured with tumor cell lines determined by qRT-PCR. $n=5$, ${ }^{*} P<0.05$ relative to untreated, ${ }^{\dagger} P<0.05$ relative to M1-induced. Data are mean \pm SEM; $P$ values calculated by 2-tailed Student's $t$ test.

to almost naive levels, while prostate cancers showed no statistically significant suppression (Figure 1E), though each cancer cell line expressed Pros1 (Supplemental Figure 1D). Clearly, Pros1 may be of critical importance to human tumor immune suppression, though its role in individual tumor types requires further study.

To determine whether the M1 suppression in coculture was solely reliant on tumor-secreted Pros1, we used CRISPR tech- nology to functionally delete the Pros1 gene in the B16F10 cells (designated BdP cells). We induced a 2-bp frame-altering deletion in the $\mathrm{N}^{\prime}$ terminal-most EGF-like domain, which we validated by sequencing (Supplemental Figure 7, A and B). Quantitative reverse transcription PCR (qRT-PCR) measurement of transcripts 5 ' to the deletion site showed a $75 \%$ reduction in the truncated transcription product, which if translated is incapable of binding TAM 

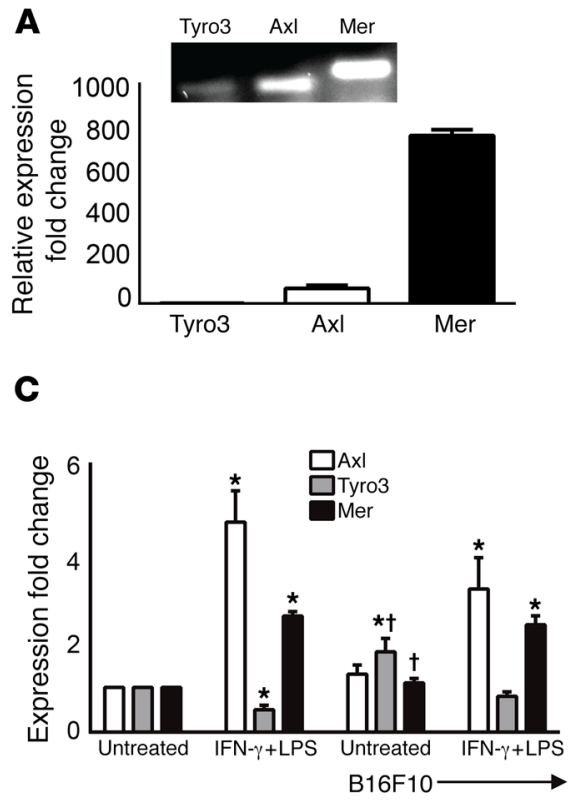

B

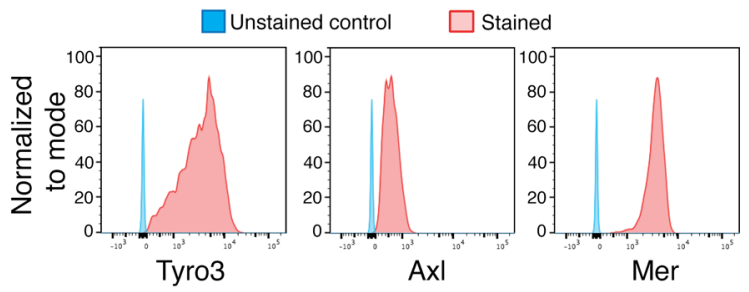

D

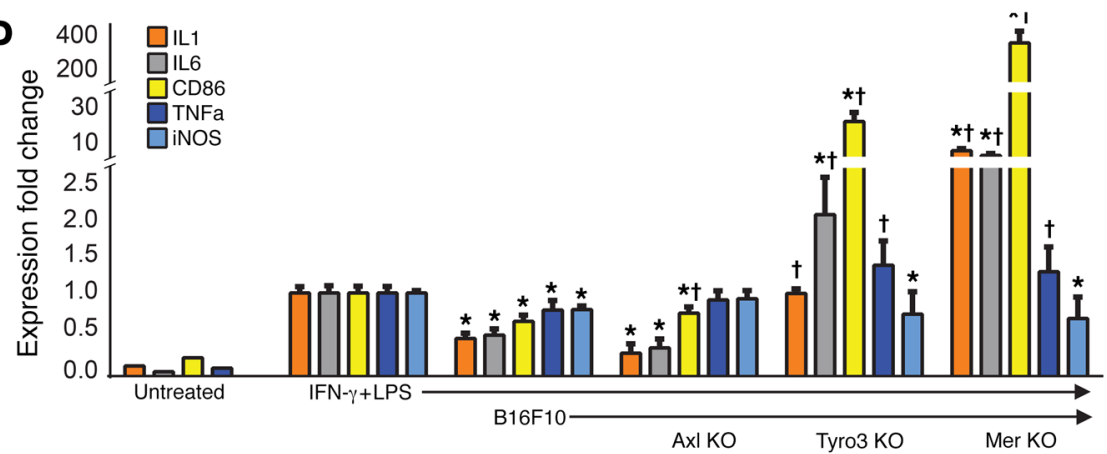

Figure 2. Tumor-secreted Pros1 inhibits M1 polarization through Mer and Tyro3. Expression analysis of Tyro3, Axl, and Mer in peritoneal macrophages by RT-PCR and qRT-PCR $(\mathbf{A} ; n=4)$ and flow cytometry (B, $n=3)$. (C) qRT-PCR analysis of TAM expression levels in M1-induced macrophages cultured in the presence or absence of B16F10 cells. $n=4,{ }^{*} P<0.05$ relative to untreated, ${ }^{+} P<0.05$ relative to M1-induced, 2 independent replicates. (D) Pros1-mediated suppression of M1-associated gene expression in Axl- but not Tyro3- or Mer-KO macrophages. $n=8,{ }^{*} P<0.05$ relative to M1-induced, ${ }^{\dagger} P<0.05$ relative to M1-induced macrophages cocultured with B16F10 cells, 2 independent replicates. Data are mean \pm SEM; $P$ values calculated by 2 -tailed Student's $t$ test.

receptors (Supplemental Figure 7C). The growth rate of BdP cells relative to B16F10 cells was unaffected by the loss of Pros1 as measured by MTT assay, nor did addition of Pros1 to the BdP cell line increase its growth rate (Supplemental Figure 7D). Thus, in these cells Pros1 is not an autocrine growth factor. However, the ability to suppress M1 polarization through paracrine signaling was lost in BdP cells cocultured with macrophages, showing that tumorsecreted Pros1 was necessary and sufficient to inhibit macrophage M1 activation (Figure 1D).

Pros1 signaling occurs through macrophage Mer and Tyro3 receptors. Mer is expressed in all macrophage subsets regardless of their bone marrow or other developmental origin. Tissue-specific macrophages can also express other TAM family members, and each may have tissue-selective roles. In peritoneal macrophages we observed that all 3 TAM receptors were expressed (Figure 2A), similar to previously published findings for BMDMs, with the exception of Tyro3 (20). Mer was the most abundantly transcribed of the 3 receptors, with approximately 700-fold higher mRNA levels compared with Tyro3 and 11-fold higher than Axl (Figure 2A). Flow cytometric analysis showed that the peritoneal macrophages expressed the 3 TAM receptors on their surface (Figure 2B). Unlike Mer and Axl, which were uniformly expressed, the level of Tyro3 expression varied within the macrophage population (Figure 2B). Our results suggest that while Tyro 3 is not transcribed as robustly as Mer, Tyro3 protein is nevertheless present at some level on resident mouse peritoneal macrophages under our conditions of isolation and study (see Methods). To further understand the effects of IFN- $\gamma$ and LPS on TAM surface expression, we characterized M1-polarized macrophages by flow cytometry. While we noted some differences in relative expression compared with qRT-PCR, we observed increases in Mer and Tyro3 surface protein (Supple- mental Figure 8). The RNA/protein discrepancy may be attributable to differential TAM receptor turnover.

To determine whether tumor-secreted factors can alter the expression of TAM receptors on macrophages, we utilized our Transwell coculture system. We treated macrophages with IFN- $\gamma$ and LPS in the presence or absence of B16F10 cells. We observed that, similar to BMDMs (20), M1-polarized macrophages increased Axl expression 4.7-fold, though we also observed a 2.4-fold increase in Mer expression (Figure 2C). We did not find a statistically significant difference in TAM RNA expression when M1-polarized macrophages were cocultured with the tumor cells (Figure 2C).

To identify the role of individual TAM receptors in M1 suppression, we isolated peritoneal macrophages from Axl- (21), Tyro3(21), and Mer-KO (8) mice and stimulated them with IFN- $\gamma$ and LPS in the presence or absence of B16F10 cells. Prior to doing so, we assayed whether macrophages from individual TAM-KO mice expressed their respective receptors by both qRT-PCR and flow cytometry (Supplemental Figure 9, A and B, respectively). The targeted knockouts reduced the individual macrophage TAMs, and loss of single receptors often affected the relative expression of the other receptors, presumably a compensatory mechanism.

With respect to function, Axl-KO M1-induced macrophages failed to eliminate the suppression induced by coculture with B16F10 cells (Figure 2D), indicating that in this population, Axl, which does not bind Pros1, does not play a role. In contrast, when cocultured with B16F10 cells neither Tyro3- nor Mer-KO macrophages demonstrated Pros1-mediated M1 suppression (Figure 2D). First, these findings support data indicating that Pros1 binds to Tyro3 and Mer but not Axl (12). Second, loss of either Mer or Tyro3, which is not as highly transcribed, abrogated M1 suppression. The necessity of both RTKs suggests either heterodimeriza- 

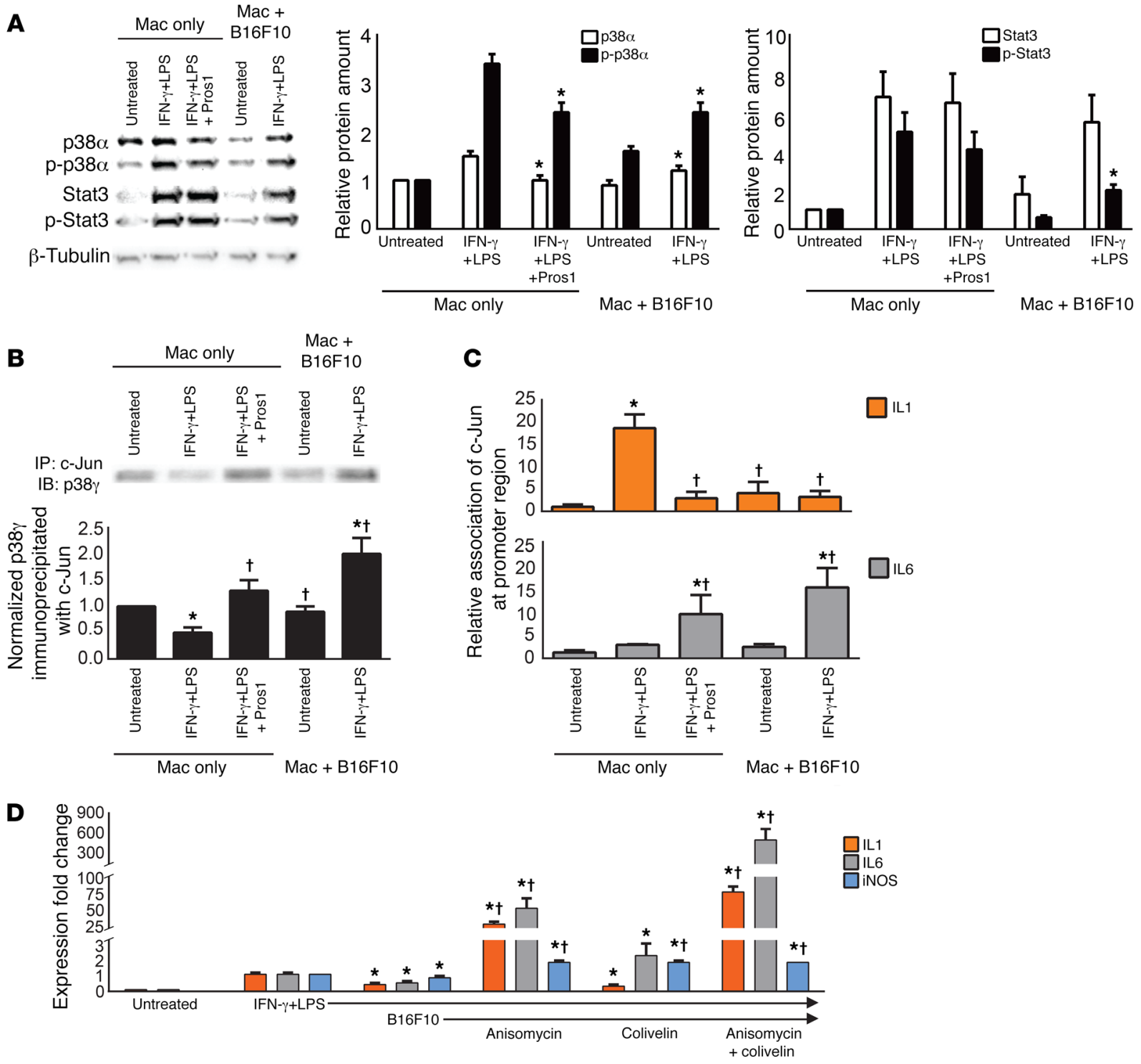

Figure 3. Pros1 inhibits M1 activity by inactivating p38 $\alpha$. (A) Western blot analysis of p38 $\alpha, p-p 38 \alpha$, Stat3, and p-Stat3 in M1-induced macrophages (Mac) treated with Pros1 or cocultured with B16F10 cells. $n=4,{ }^{*} P<0.05$ relative to M1-induced, 3 independent replicates. (B) Coimmunoprecipitation of $p 38 \gamma$ with c-Jun in M1-polarized macrophages treated with Pros1 or cocultured with B16F10 cells. $n=4,{ }^{*} P<0.05$ relative to M1-induced, ${ }^{t} P<0.05$ relative to B16F10 suppressed, 2 independent replicates. (C) qRT-PCR of IL1 and IL6 promoter regions following c-Jun ChIP. $n=5$, ${ }^{*} P<0.05$ relative to macrophageonly untreated, ${ }^{\dagger} P<0.05$ relative to macrophage-only IFN- $\gamma+$ LPS treatment, 2 independent replicates. (D) Immunosuppression of M1-induced macrophages cocultured with B16F10 cells is reversed after treatment with anisomycin and colivelin. $n=4,{ }^{*} P<0.05$ relative to M1-induced, ${ }^{\dagger} P<0.05$ relative to B16F10 suppressed. Data are mean \pm SEM; $P$ values calculated by 2-tailed Student's $t$ test.

tion (22) or independent but mutually necessary signals. To test the former, we cocultured M1-induced macrophages with B16F10 cells and immunoprecipitated using a Mer-specific antibody. We did not detect coimmunoprecipitated Tyro3 (data not shown). However, with the lower abundance of Tyro3, we cannot rule out heterodimerization. Nonetheless, these data clearly indicate that absence of either Mer or Tyro3 abrogates the response, suggesting the need for oligomers or heterodimers to amplify the signal, nonredundant but necessary individual receptor signals, or both.

We also note that in the absence of Tyro3 or particularly Mer, the induction of M1 genes IL1, IL6, and CD86 was strikingly higher. There did appear to be selectivity, with IL1 and IL6 being restored to the non-suppressed state in the Tyro3-deleted mac- rophages and being 5.1-fold higher in the absence of Mer. This could be interpreted as a loss of tonic inhibition (as occurred in our originally observed inflammatory response in the LPS-treated Mer-KO mouse; ref. 8), allowing an increased transcription of these genes, though TAM-KO macrophages did not appear to be hyperresponsive to IFN- $\gamma$ and LPS treatment in the absence of B16F10 coculture (Supplemental Figure 10). The effect may also reflect the secretion of other factors from B16F10 cells that can amplify the M1 response but were held in check by Pros1 Mer/ Tyro3 action. This putative hyper-responsiveness was not global, as iNOS levels were unaffected.

Pros 1 inhibits p38 signaling in M1-induced macrophages. TAM activation influences multiple intracellular signaling pathways, 

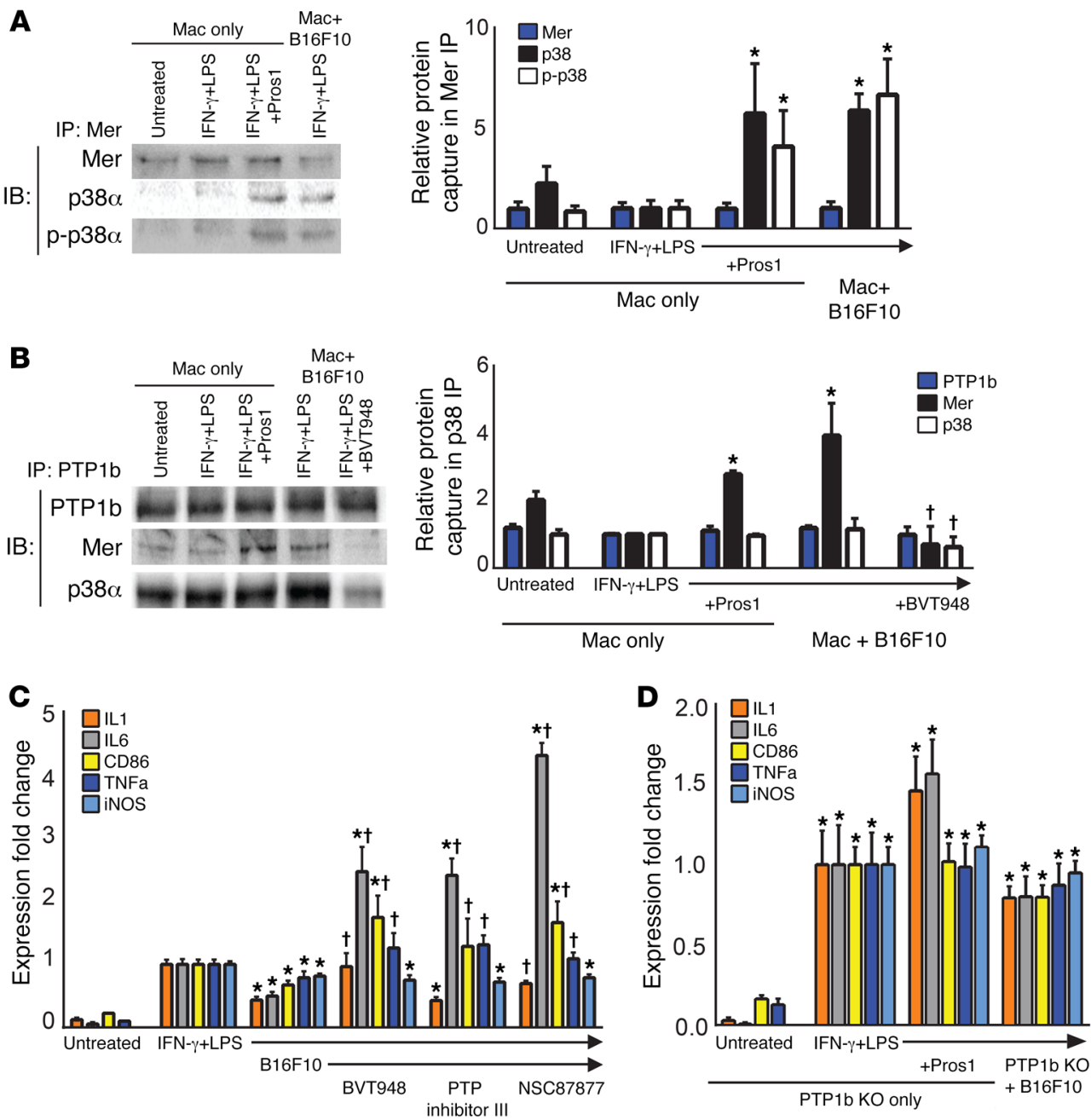

Figure 4. Mer complexes with PTP1b and inhibits p38-mediated M1 gene expression. (A) Coimmunoprecipitation of $p 38 \alpha$ and $p-p 38 \alpha$ with Mer after stimulation of $\mathrm{M} 1$-induced macrophages with Pros1. $n=3$, ${ }^{*} P<0.05$ relative to macrophage-only IFN- $\gamma+$ LPS treatment, 3 independent replicates. (B) Coimmunoprecipitation of Mer and p38 $\alpha$ with PTP1b after stimulation of M1-induced macrophages with Pros1 and/or BVT948. $n=3,{ }^{*} P<0.05$ relative to macrophage-only IFN $-\gamma+$ LPS treatment, ${ }^{+} P<0.05$ relative to IFN $-\gamma+$ LPS-treated B16F10 cocultured macrophages, 3 independent replicates. (C) Expression of M1-associated genes in M1-induced macrophages cocultured with B16F10 in the presence or absence of PTP inhibitors BVT948, PTP inhibitor III, and NSC87877. $n=8,{ }^{*} P<0.05$ relative to M1-induced macrophages, ${ }^{\dagger} P<0.05$ relative to $\mathrm{M} 1$-induced macrophages cocultured with B16F10 cells. (D) M1-induced PTP1b-KO macrophages cultured in the presence of Pros1 or cocultured with B16F10 cells. $n=4,{ }^{*} P<0.05$ relative to $M 1$-induced macrophages. Data are mean $\pm \mathrm{SEM}$; $P$ values calculated by 2 -tailed Student's $t$ test. as reviewed by Graham et al. (23). An initial screen of several pathways showed that Pros1, either exogenous or derived from cocultured B16F10 cells, did not substantially affect phosphorylation or total protein levels of key signaling transducers in macrophages (Supplemental Figure 11). Of particular interest, however, is the p38 MAP kinase pathway, which has been previously identified as a key signaling transducer in the proinflammatory response of macrophages $(24,25)$. In a DC model, TAM signaling reduced p38 phosphorylation, which was associated with an inhibited inflammatory response (26). We therefore examined M1-induced macrophages for changes in p38 $\alpha$ and showed a $30 \%$ decrease in p-p38 $\alpha$ levels in the presence of Pros1 or B16F10 cells, as well as small but statistically significant changes in total p38 $\alpha$ 24 hours after treatment (Figure 3A). We observed a similar suppression in total p38 $\alpha$ and p-p38 levels as early as 2 hours after treatment (Supplemental Figure 12).

Stat 1 has been described as a downstream effector of TAM signaling in DCs (26). We first assayed whether Stat inhibition would alter M1-associated gene expression. When M1-induced macrophages were treated with the Stat inhibitors fludarabine (Stat1) (27), S3I-201 (Stat3) (28), and Stat 5 inhibitor (Stat5) (29), we observed that Stat 3 inhibition completely ablated expression of iNOS but not other M1 genes (Supplemental Figure 13), while other Stat inhibi- tors had only minor effects (data not shown). We also found that Jak1/2 inhibition with baricitinib (30) treatment similarly ablated iNOS expression in M1 macrophages (Supplemental Figure 13) but left other M1-associated gene expression largely unaffected (data not shown). We next examined total and p-Stat3 levels. Treatment with exogenous Pros1 did not significantly change total or p-Stat3, though coculture with Pros1-secreting B16F10 cells led to a 59\% decrease in p-Stat3 (Figure 3A). The latter could be attributable to other tumor-secreted proteins or alternative effects on phosphorylation of exogenous versus native Pros1. Neither Pros1 addition nor B16F10 coculture affected Stat3 or p38 transcription (Supplemental Figure 14), showing that Pros1 signaling affects each signaling protein posttranslationally.

p38 has been linked to the transcription of IL1, IL6, and TNFa (31), the expression of all of which was reduced by the presence of Pros1 in our assays. Conversely, iNOS expression, which was not reduced in M1-induced macrophages treated with Pros1, is governed by Jak/Stat3 signaling (see above). We next focused on how Pros1 signaling affects p38 and c-Jun, a component of the AP1 transcription complex known to bind to p38 and activate transcription of M1 genes, including IL1 and IL6 (32). We performed coimmunoprecipitation of c-Jun on M1-induced macrophages in the presence or absence of Pros1 or B16F10 cells. Pros1 increased 
A

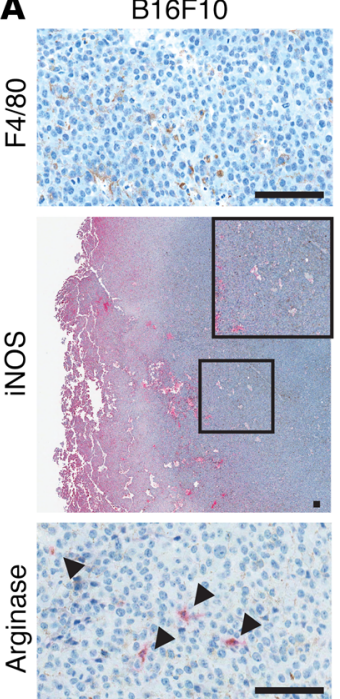

B
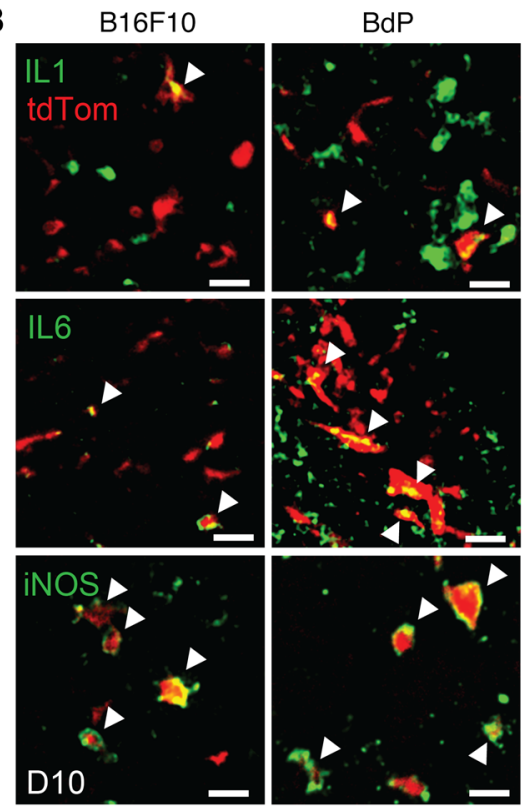

IL1

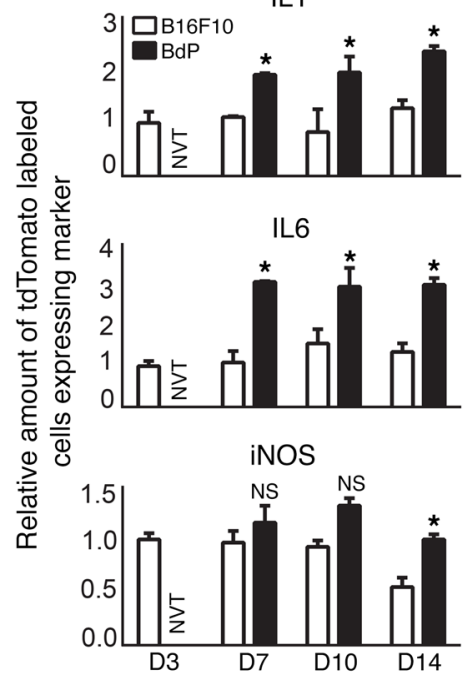

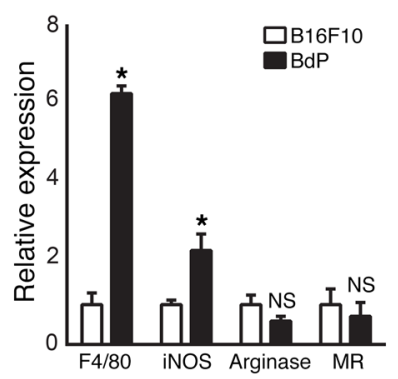

C
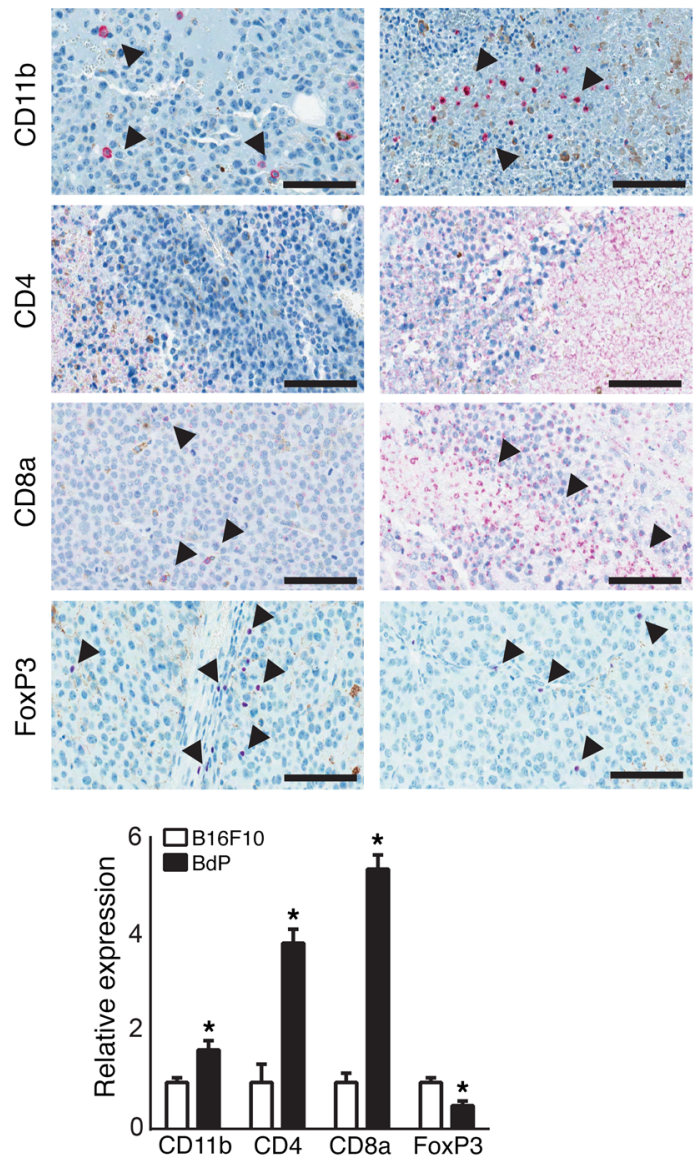

Figure 5. Pros1 inhibits M1 polarization and reduces immune infiltrate in vivo. (A) Immunohistochemical staining of macrophage markers in B16F10 and $\mathrm{BdP}$ endpoint tumors of $\mathrm{C} 57 \mathrm{BL} / 6$ mice. (B) Immunofluorescence staining of M1 markers in B16F10 or BdP tumors in Lyz2-Cre:tdTomato mice 3, 7,10 , or 14 days after implantation. NVT, no visible tumor. (C) Immunohistochemical staining of immune infiltrate markers in B16F10 or BdP endpoint tumors in $\mathrm{C} 57 \mathrm{BL} / 6$ mice. Scale bars: $100 \mu \mathrm{m}$ (insets, $1,200 \mu \mathrm{m}$ square) in $\mathbf{A}$ and $\mathbf{C}, 20 \mu \mathrm{m}$ in $\mathbf{B} ; n=4$ for all, ${ }^{*} P<0.05$ relative to B16F10 tumor. Data are mean $\pm \mathrm{SEM} ; P$ values calculated by 2-tailed Student's $t$ test. 

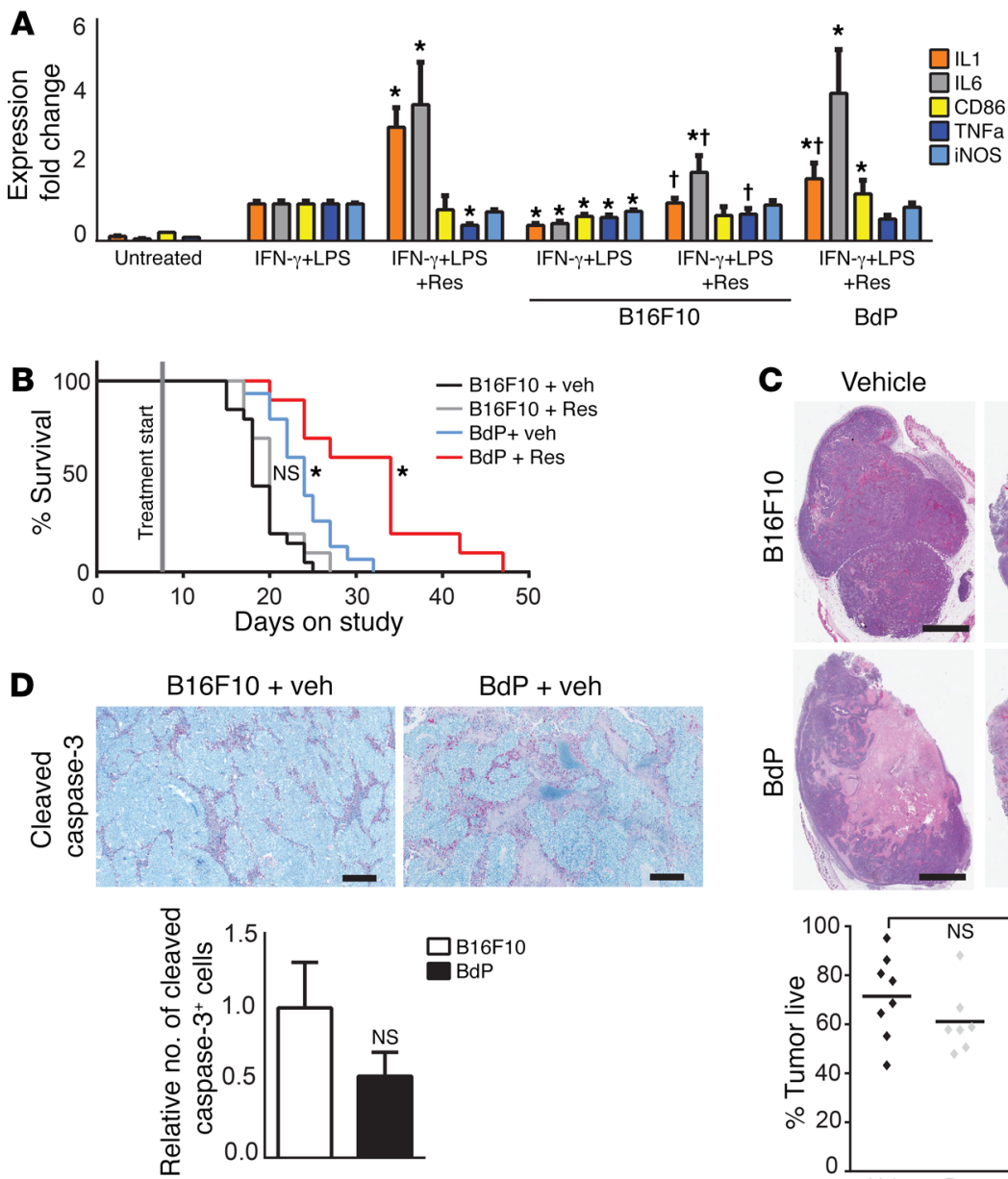
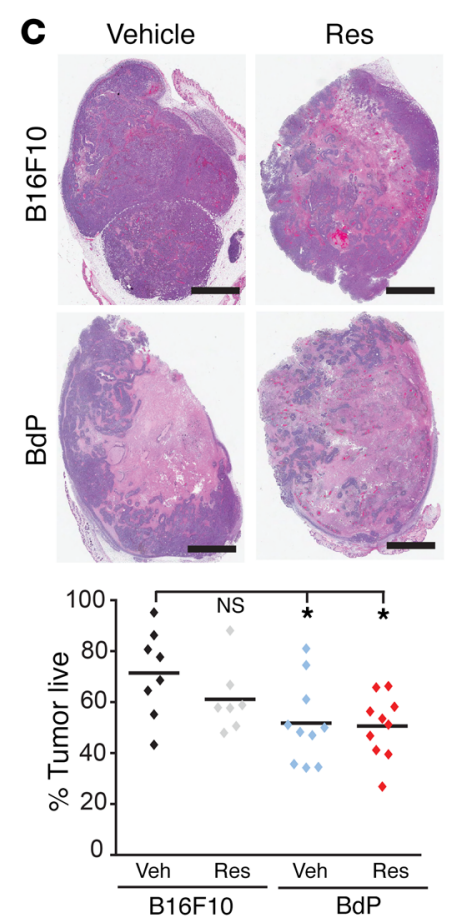

Figure 6. Tumors lacking Pros1 are more susceptible to TLR-based therapy. (A) Gene expression of M1-induced macrophages treated with resiquimod (Res) cultured in the presence or absence of B16F10 and BdP cells. $n=8,{ }^{*} P<0.05$ relative to IFN- $\gamma+$ LPS, ${ }^{\dagger} P<0.05$ relative to IFN $-\gamma+\mathrm{LPS}+$ resiquimod, 2 independent replicates. (B) Survival of $C 57 \mathrm{BL} / 6$ mice bearing B16F10 or BdP tumors with vehicle (saline) or resiquimod treatment. $n=10$, ${ }^{*} P<0.05$ relative to vehicle-treated B16F10, 2 independent replicates. (C) H\&E stain of tumors isolated from B16F10 or BdP tumors treated with vehicle or resiquimod, and quantitation of percent viable tumor. Scale bars: $3 \mathrm{~mm}, n=7-10,{ }^{*} P$ $<0.05$ relative to vehicle-treated B16F10, 2 independent replicates. (D) Immunohistochemical staining of tumor sections for the apoptosis marker cleaved caspase-3 and quantitation relative to B16F10. Scale bars: $100 \mu \mathrm{m}, n=5,2$ independent replicates. Data are mean \pm SEM; $P$ values calculated by 2 -tailed Student's $t$ test. the association of the inhibitory isoform of p38, p38 $\gamma$, with c-Jun (Figure 3B), which has been shown to reduce M1 gene transcription (32). ChIP comparing untreated and Pros1-suppressed M1 macrophages demonstrated a 9-fold reduction in c-Jun with the IL1 promoter (Figure 3C). Interestingly, association of c-Jun with the IL6 promoter region was increased 6-fold in the presence of Pros1 (Figure 3C). Our data suggest alterative modalities of suppression; the IL1 promoter has decreased c-Jun binding, and the IL6 promoter has increased binding of an inhibitory complex.

To confirm that p38 was a downstream effector of Pros1/TAM signaling, we used a gain-of-function approach and added the p38 activator anisomycin (33) to naive, M1-induced, and Transwellcocultured M1-induced macrophages (Figure 3D and Supplemental Figure 15). In all cases, anisomycin treatment increased IL1 and IL6 expression, though M1-induced macrophages showed a greater expression increase than did naive macrophages. We also observed that not only was B16F10-mediated suppression eliminated, but expression of IL1 and IL6 was increased 11- and 41-fold, respectively, relative to M1-induced macrophages (Figure 3D). We also observed a 2-fold increase in iNOS (Figure 3D), which suggests some crosstalk with the Jak/Stat3 pathway. To determine the effect of Stat 3 activation on the restoration of M1 gene expression, we added colivelin (34) to cocultured M1 macrophages and observed a 2-fold increase in iNOS and IL6 expression, while
IL1 expression was unaffected (Figure 3D). Exploring the idea of crosstalk between the p38 and Stat3 pathways, we treated M1 macrophages cocultured with B16F10 cells with anisomycin and colivelin, and observed synergistic activation of M1 gene expression, with increases of 70-, 478-, and 2-fold for IL1, IL6, and iNOS, respectively (Figure 3D). While these drugs may well have effects on alternative stress response pathways (such as MAPK), the combination suggests some form of synergistic activity between the two pathways. Taken together, these findings suggest that Pros1/ TAM signaling primarily reduces aspects of M1 activation by reducing $\mathrm{p} 38 \alpha$ function and altering c-Jun promoter interaction.

Mer activation inhibits 338 activity through a PTP1b:Mer complex. A direct link between TAM receptors and p38 signaling has not to our knowledge been previously established. Using coimmunoprecipitation, we explored whether $\mathrm{p} 38$ complexes with Mer upon ligand activation and showed that Pros1-treated or B16F10cocultured M1 macrophages exhibited a 5.8-fold-increased p38 $\alpha$ association with Mer (Figure 4A).

Structurally and biochemically, Mer does not appear to have motifs for direct interaction with p38. However, published findings indicated that PTP1b is a negative regulator of macrophage inflammatory response $(35,36)$ and directly interacts with p38, reducing phosphorylation and downstream activity (37). PTP1b has also been shown to be critical in facilitating IFN- $\alpha / \beta$ receptor- 

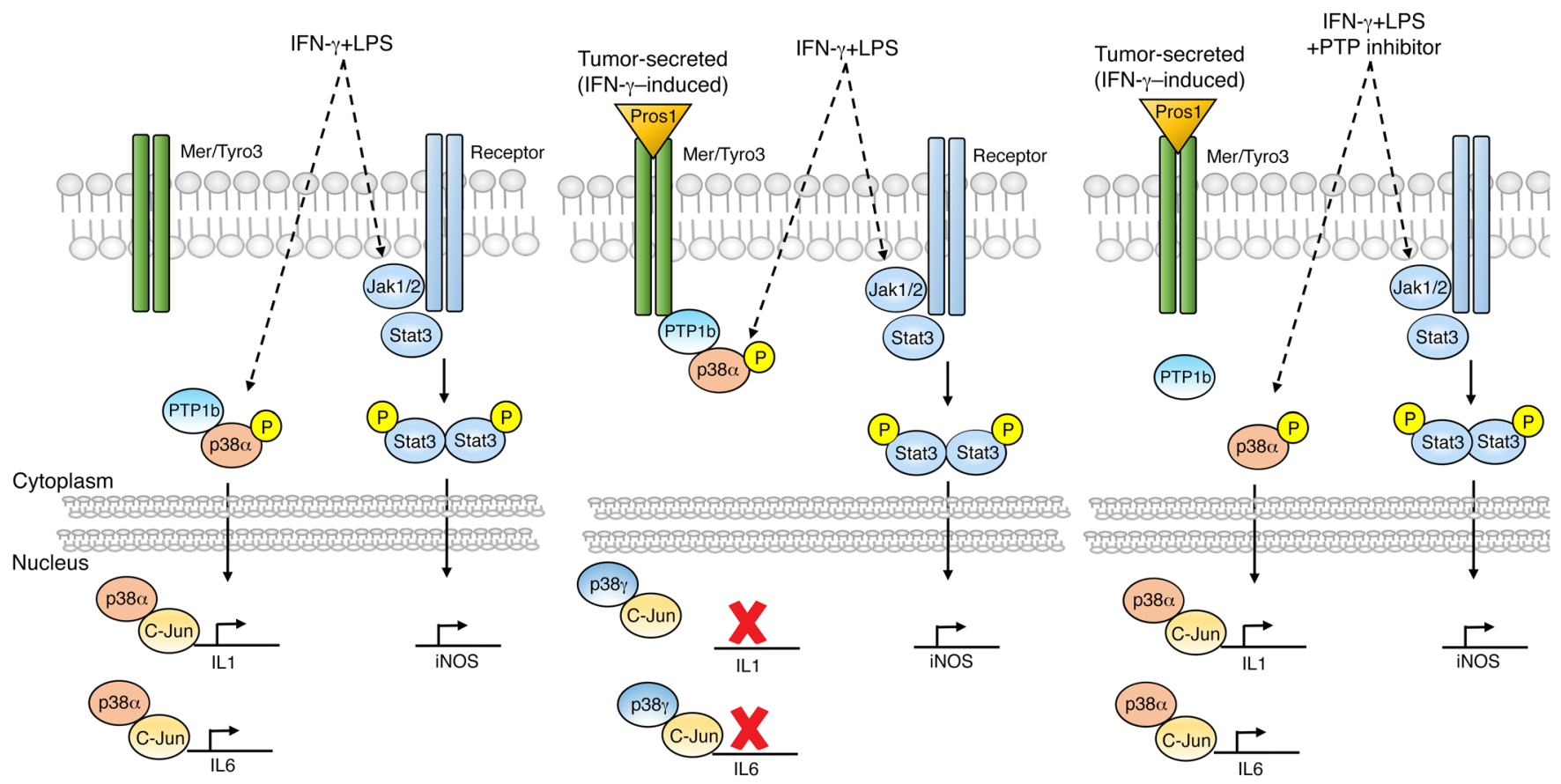

Figure 7. Tumor-secreted Pros1 inhibition of M1-associated gene expression in a Mer/Tyro3-dependent manner. Proposed signaling model. Left: IFN- $\gamma+$ LPS stimulate transcription of macrophage M1 cytokines IL1 and IL6 in a p38a-dependent manner. Middle: Tumor-derived Pros1 initiates Mer-dependent ternary complex formation, sequestering $\mathrm{p} 38 \mathrm{a}$ and suppressing IL1 and IL6 transcription by alternative mechanisms. Right: p38a sequestration is reversed in the presence of PTP1b inhibitors, and M1 gene expression is restored.

mediated (IFNAR-mediated) endocytosis (38), and Axl complexes with IFNAR when stimulated with Gas6 (26). Interestingly, PTP1b-KO mice also show reduced tumor progression and metastasis in murine mammary tumor models $(39,40)$, comparable to our findings with Mer-KO mice (13). We asked whether Pros1 may elicit ternary complex formation consisting of Mer, IFNAR, and PTP1b to modulate p38 activity. Immunoprecipitation of PTP1b from M1-stimulated macrophages demonstrated that association of PTP1b with Mer increased by 2.7- and 3.9-fold in the presence of Pros1- and B16F10-cocultured cells, respectively (Figure 4B). We did not observe IFNAR in the complex (data not shown). In addition, PTP1b was constitutively bound to p38 $\alpha$ (Figure 4B). However, when M1 macrophages cocultured with B16F10 cells were treated with the PTP inhibitor BVT948 (41), association of PTP1b with Mer was nearly eliminated and the binding of p38 $\alpha$ reduced (Figure 4B). We observed a similar response with other PTP inhibitors such as PTP inhibitor III (42) (data not shown).

We hypothesized that a drug-induced allosteric change in PTP1b structure in the Mer/PTP1b/p38 $\alpha$ complex (see above) may relieve Pros1-mediated M1 suppression. We used our coculture assay and determined that addition of the PTP inhibitors BVT948, PTP inhibitor III, and NSC87877 (43) alleviated the suppression and restored cytokine-induced M1 polarization (Figure 4C), though by themselves the inhibitors did not induce a significant M1 response in naive macrophages or positively affect M1 gene expression in M1-induced macrophages (Supplemental Figure 16). To test a more definitive genetic approach, we isolated peritoneal macrophages from PTP1b-KO mice. Treatment of cytokinestimulated, M1-induced PTP1b-KO macrophages with exogenous Pros1 or in Transwell coculture with B16F10 cells failed to show any statistically significant change in M1-associated gene expression (Figure 4D). This abrogation of the Pros1/B16F10-suppressive effect in PTP1b-KO macrophages specifically implicates the Mer/PTP1b complex as necessary for Pros1-, Mer/Tyro3-dependent M1 suppression. Thus, both genetic and pharmacologic perturbation abrogated Pros1-dependent suppression (Figure 4, C and D), reinforcing the functional role of PTP1b activity in Pros1based immune suppression.

Pros1 suppresses the immune response in vivo. After observing that Pros1 effectively inhibits macrophage M1 polarization in vitro, we tested whether paracrine Pros1 secretion had a similarly suppressive effect in vivo. To do so we implanted the BdP or B16F10 cell line in $\mathrm{C} 57 \mathrm{BL} / 6 \mathrm{~J}$ mice and harvested tumors when they reached $1.5 \mathrm{~cm}$ in any direction. Tumors were then sectioned, followed by immunohistochemical staining for common macrophage markers. We observed a 6-fold increase in the number of F4/80-positive macrophages in BdP tumors relative to parental B16F10 tumors (Figure 5A). Our in vitro findings were recapitulated in that the number of cells expressing the M2-associated genes arginase and MR was not significantly increased (Figure 5A), further demonstrating that paracrine Pros1 does not skew macrophages all the way toward an M2 phenotype in this model. We also observed a 2 -fold increase in the number of cells expressing iNOS (Figure $5 \mathrm{~A})$. To determine whether there was a difference in macrophage TAM receptor expression in the 2 tumor models, we conduced flow cytometry on B16F10 and BdP tumors harvested 14 days after implantation. We found that in $\mathrm{F} 4 / 80$-positive intratumoral macrophages, Tyro3 expression was almost doubled in BdP tumors relative to B16F10 tumors, while differences in Axl or Mer expression were not statistically significant (Supplemental Figure 17). 
Because of the complex tumor milieu, which comprises a variety of immune cell types, we utilized genetic labeling to identify macrophages within the tumor. We crossed mice bearing lysozyme 2-Cre (Lyz2-Cre) (44) with lox-Stop-lox tdTomato reporter mice (45). The progeny mice have macrophages labeled with the tdTomato reporter. Peritoneal macrophages isolated from Lyz2-Cre:tdTomato mice showed greater than 99\% labeling efficiency when immunostained for the macrophage marker F4/80 (Supplemental Figure 18A) and displayed characteristic phenotypic changes and increased M1 marker expression (iNOS and IL1) when treated with IFN- $\gamma$ and LPS (Supplemental Figure 18B). Immunostaining of intratumoral tdTomato cells showed that approximately $40 \%$ were $\mathrm{F} 4 / 80$ positive (Supplemental Figure 18C), which is consistent with F4/80 macrophage labeling in vivo. To determine whether there were dynamic changes in macrophage M1 gene expression over time, we implanted $1 \times 10^{5} \mathrm{~B} 16 \mathrm{~F} 10$ or BdP cells and harvested resultant tumors 3,7 , 10 , or 14 days later. Immunofluorescence staining was then performed, and sections were imaged by confocal microscopy. We determined the percentage of tdTomato-labeled macrophages expressing IL1, IL6, or iNOS. While BdP tumors were not observable at the 3-day time point, at 7 days we did observe a 2 -fold and 3 -fold increase in the number of macrophages expressing IL1 and IL6, respectively, in BdP tumors compared with B16F10 tumors. The percentage of iNOS-expressing macrophages did not differ significantly until 14 days after implantation (Figure 5B). These findings recapitulate our in vitro observations that Pros 1 reduces p38 $\alpha$-driven IL1 and IL6 expression, leaving Jak/Stat3-driven iNOS expression relatively unaffected.

With the increased macrophage infiltration and M1 polarization, we next examined infiltration by other immune cells in B16F10 or BdP tumors by performing immunohistochemical staining on tumor sections. The number of CD11b-positive cells almost doubled, while the population of CD4-positive cells increased 4 -fold (Figure 5C). The number of CD8a-positive T cells increased 5 -fold, while the number of regulatory $\mathrm{T}$ cells was reduced by $50 \%$, as shown by FoxP3 staining (Figure $5 \mathrm{C}$ ). While a previous study showed that Pros1 can induce proliferation in T cells (46), the loss of tumor-secreted Pros1 was apparently outweighed by increased innate immune activation to yield an increased number of intratumoral T cells. Taken together, our findings demonstrate that tumorderived Pros1 effectively dampens the innate immune response, as well as the consequent adaptive immune response. Elimination of tumor cell Pros1 appears to substantially mitigate the suppressive microenvironment, promoting adaptive cell infiltration.

Pros1-deficient tumors are more susceptible to immune-stimulatory therapy. When DCs were studied by others, TAM RTK activation was shown to inhibit an array of MyD88-dependent and -independent TLR agonists (26). We also observed that M1 transcriptional activation across the spectrum of TLRs (including TLR2, -3, -4, -5, -6, -7, -8) was reduced when peritoneal macrophages were cocultured with B16F10 cells (data not shown). To study M1-associated gene expression in B16F10 suppressed macrophages, we first treated macrophages alone with resiquimod, an agonist of TLR7/8 (47). We observed additional 3- and 5-fold increases in IL1 and IL6 gene expression, respectively, when IFN- $\gamma$ and LPS-induced macrophages were treated with resiquimod for
24 hours (Figure 6A). Expression of other M1 genes was either not significantly increased (CD86 or iNOS) or reduced (TNFa) (Figure 6A). When M1-stimulated macrophages were next treated with resiquimod in the presence of Pros1-expressing B16F10 cells, agonist-dependent M1 gene expression was again suppressed, with IL1 and IL6 reduced by $67 \%$ and $64 \%$, respectively (Figure 6A). However, in resiquimod-treated M1 macrophages cocultured with Pros1-deficient B16F10 cells, M1 gene expression was largely restored (Figure 6A), strongly suggesting that tumor cell-derived paracrine Pros1 can suppress both cytokine- and TLR-stimulated M1 gene expression.

We hypothesized that without tumor-secreted Pros1 to suppress M1 polarization, TLR-based stimulatory immunotherapy would be more effective in eliciting an antitumor response. To test this, we implanted parental or Pros1-deleted B16F10 cells in C57BL/6J mice and treated them with vehicle or resiquimod intraperitoneally. We noted a $30 \%$ increase in survival duration with vehicle-treated BdP tumors as compared with vehicle-treated B16F10 tumors (Figure $6 \mathrm{~B})$. When treated with resiquimod, B16F10 tumor-bearing mice did not show a significant increase in survival (Figure 6B). However, when BdP tumor-bearing mice were treated with resiquimod, survival was almost doubled (Figure 6B).

Based on our observations that Pros1-deficient tumors showed increases in both macrophage M1 polarization and overall immune infiltrate (Figure 5), we examined tumor sections with H\&E staining to determine whether there was an effect on tumor cell viability. We saw a significant decrease in the percentage of viable tumor in the Pros1-deficient tumors relative to Pros1-secreting tumors (Figure 6C). Resiquimod treatment of B16F10 tumors did not significantly reduce tumor viability or increase longevity, though treatment of Pros1-deficient tumors resulted in both reduced tumor viability and prolonged survival (Figure 6, B and C). While resiquimod treatment of Pros1-deficient tumors did not eliminate the cancer prior to reaching Division of Laboratory Animal Medicine-mandated size restrictions, this may be in part due to the inability of the host to resolve the nonviable tumor, which effectively added to the overall tumor mass.

To understand whether differences in viability were the result of tumor cell apoptosis or increased tumor cell killing via increased proinflammatory cell infiltrate, we performed immunohistochemical staining on tumor sections for the apoptosis marker cleaved caspase-3. We did not observe a statistically significant difference in cleaved caspase-3 levels between parental and Pros1deficient tumors at the time of tumor harvest (Figure 6D). Therefore, cell viability was reduced by either necrosis or apoptosis that was no longer detectable at the time of harvest, possibly because of an increased rate of efferocytosis due to an increased number of phagocytic cells within the tumor (Figure 5A). Since the TLR agonist was administered systemically, it seems likely that the increase in survival and reduction in live tumor cell mass occurred through innate and adaptive tumor-specific immune responses enhanced by the removal of paracrine Pros1.

With respect to the clinical applicability of these findings, we examined the overall pattern of Pros1 expression in multiple tumor types analyzed by The Cancer Genome Atlas (TCGA); there was a wide range of Pros1 RNA expression, suggesting that a proportion of multiple tumor types may exhibit Pros1 levels contribut- 
ing to an immunosuppressive microenvironment. And while there were instances of mutation, the predominant expression appeared to be in nonmutant RNA (Supplemental Figure 19A). Interestingly, there do seem to be several tumor types demonstrating frequent amplification, in addition to mutation, that may confer a Pros1 gain-of-function phenotype, benefiting tumor progression through suppression of the innate immune response (Supplemental Figure 19B). However, bioinformatics analysis of TCGA data did not reveal a prognostic effect in specific tumors; additional analysis of subsetted therapy responders and nonresponders may be necessary to tease out predictive effects of Pros1 expression.

\section{Discussion}

This report demonstrates a mechanism by which cancer cells inhibit the antitumor immune response by mimicking a physiologic innate immune process - a form of innate immune checkpoint activity induced by paracrine secretion from the tumor cell. The immune system is, and needs to be, finely balanced to detect and eliminate foreign pathogens yet not over- or chronically react, causing excessive damage to the host. Lack of physiologic antiinflammatory responses can have adverse effects ranging from chronic autoimmune disease to death from septic shock. Multiple investigators have now confirmed the original demonstration of Mer suppression of inflammatory signaling (8) by defining the antiinflammatory nature of TAM RTK signals in multiple tissues and across innate immune cell types (11). A multiplicity of TAM RTK actions preserve immunological homeostasis. In this report we show that tumor cells themselves can subvert this mechanism by creating an immune suppressive environment through paracrine secretion of TAM ligand. Tumor-secreted Pros1 can bind to Mer and Tyro3 receptors on resident and elicited macrophages and effectively suppress their ability to adopt an M1, antitumor phenotype.

Tumor expression of Pros1, as an immune cloaking device, might be gained through gene amplification or perhaps gain-offunction mutation (Supplemental Figure 19), but it is more likely that the wide range of tumor Pros1 expression is induced, for example by IFN- $\gamma$. The IFN- $\gamma$ responsiveness of Pros1 transcription across tumor types, similar to that of PD-L1 or indoleamine2,3-dioxygenase (IDO) (48), may suggest that Pros1 induction is a broad mechanism by which tissues dampen the innate response to immune activation. Others have reported that $\mathrm{T}$ cell activation itself can also upregulate Pros1, with the consequence of paracrine feedback to suppress DC function (49). We add tumor cell Pros1 to what may be a broader IFN- $\gamma$-triggered "immune defensive" transcriptional program captured in the evolution of a neoplasm. We note that IFN- $\gamma$ did not induce Gas6, nor did the Gas6 present in B16F10 after Pros1 deletion affect these particular changes. This suggests that individual ligands may induce slightly different programs, highlighting the complexity of TAM signaling.

As others have shown, the ability of Pros1 to activate Mer and Tyro3 is clearly increased with PtdSer. However, the relatively high tumor output of Pros1, especially when exacerbated by the presence of IFN- $\gamma$, may be sufficient to overcome reduced affinity in conditions where PtdSer is limited, which is probably the case in our Transwell coculture experiments. In the in vivo tumor context, PtdSer is probably never in short supply, from apoptotic cells, tumor exosomes, and aggregating platelets or even portions of live cells, which by controlling domain flippase activity reveal patches of externalized PtdSer (50).

The downstream mechanisms affecting TAM RTK suppressive action are undoubtedly complex, tissue specific, and dependent in part on the complement of the 3 family members. In our model it is clear that both Mer and Tyro3 are involved, and not just because the ligand Pros 1 binds to both. The elimination of Tyro 3 by definitive genetic deletion abrogates the suppression in peritoneal macrophages; put another way, both Mer and Tyro3 are needed either to amplify the signal or to work via unique but mutually necessary pathways. Upon binding of tumor-secreted Pros1 to Mer, ternary complex formation comprising pre-complexed PTP1b and p38 $\alpha$ with Mer is increased. The increased complex formation correlated with decreased total p38 $\alpha$ phosphorylation, increased sequestration of $\mathrm{p} 38 \alpha$ in the Mer complex, and increased association of the inhibitory p38 isoform p38 $\gamma$ with downstream transcriptional effector c-Jun. We hypothesize that sequestration of p38 $\alpha$ in the ternary complex and/or dephosphorylation by PTP1b allows p38 $\gamma$, which is more resistant to dephosphorylation (25), to increase association with c-Jun. Examination of IL1 and IL6 promoters by ChIP demonstrated major Pros1-dependent changes in c-Jun transcription factor binding.

These results also suggest differential mechanisms of regulation for IL1 and IL6 transcription. We showed that in the case of the IL1 gene, c-Jun was 9-fold less capable of binding the IL1 promoter and initiating transcription. Alternatively, in the case of IL6, increased inhibitory complexes including c-Jun and, we suspect, p38 $\gamma$ form to suppress transcription. At this point we do not understand the details of these different promoter control mechanisms, but each correlates with reduced mRNA.

Drug-induced inhibition of PTP1b with several agents altered p38 $\alpha$ 's association with PTP1b, allowing increased p38 $\alpha$ activity or direct p38 $\alpha$ stimulation. Either mechanism would reverse Pros1-mediated immune suppression, mostly likely by destabilizing Pros1-induced Mer/PTP1b/p38 $\alpha$ ternary complex formation or overcoming p38 $\alpha$ dephosphorylation. Data in Figure 4C demonstrate that PTP1b inhibitors block Pros1-dependent suppression. Even more definitively, PTP1b-KO mouse macrophages could not respond to Pros1-mediated suppression (Figure 4D). Our model (Figure 7) summarizes how Pros1:Mer/Tyro3 signaling may dampen IFN- $\gamma /$ TLR-mediated M1-associated transcriptional activation and how it might be reversed by PTP1b inhibition. Biochemically, Mer has not been shown to have direct interaction with PTP1b. We hypothesize that Grb2, which has been demonstrated to interact with both Mer (23) and PTP1b (51), is a likely intermediary. Pharmacological inhibition of PTP1b is currently being studied as a treatment for type 2 diabetes and obesity (52). Our results suggest that PTP1b inhibition may play a role in the reactivation of the innate immune system, which could be explored as a therapeutic option.

Suppressing the p38 signaling pathway reduced expression of IL1 and IL6 in particular, but had little effect on the Stat3mediated expression of iNOS. While iNOS expression is often thought to have antitumor effects, myeloid derived suppressor cells (MDSCs) are known to inhibit the T cell response by producing ROS as well as iNOS (53). Therefore, it may be beneficial to the tumor cells to inhibit macrophage expression of some genes 
(IL1, IL6, TNFa) and not others (iNOS), thereby inhibiting both the innate and adaptive immune responses.

As Pros1 is expressed across most human tumor types (Supplemental Figure 19), continued study of tumor-mediated innate suppression through interactions with Mer/Tyro3 may elucidate novel therapeutic applications to improve tumor-specific immune response. Data from human cancer cell lines showing suppression of human macrophage line M1 cytokines (Figure 1E) suggest the phenomenon is relevant to human disease. Our data may be only part of the story, as Pros1 may have other context-specific or tumor cell growth activities as an autocrine activator of non-oncogene addiction pathways (23). Nevertheless, as we demonstrated in vivo, loss of tumor-derived Pros1 improved the function of both the innate and presumably the adaptive immune response. While the combination of Pros 1 deletion and TLR 7/8 agonists failed to "cure" the difficultto-treat B16F10, the regimen reduced viable tumor cell mass and doubled survival. The finding that IFN- $\gamma$ stimulates the production of Pros1 in B16F10 and several other cell lines (Supplemental Figure 1) suggests that this inflammatory cytokine not only increases $T$ cell suppression by inducing PD-L1 but also stimulates innate immunesuppressive mechanisms. Both of these actions are probably born from the normal yin and yang of the immune system's attempt to rid the host of pathogens without excess damage associated with chronic inflammation. Tumors invoke this intrinsic pathway or simply evolve to take advantage of this physiologic mechanism. Modalities that prevent interaction of Pros1, Mer, and Tyro3 may therefore increase the utility of immune-targeted therapies.

\section{Methods}

Generation of transgenic mice. Lyz2-Cre:R26R ${ }^{\text {tdTomato }}$ mouse lines were obtained by crossing Lyz2-Cre mice (catalog 004781, The Jackson Laboratory) with lineage reporter R26R ${ }^{\text {tdTomato }}$ mice (007908, The Jackson Laboratory). PTP1b-KO mice were obtained from the laboratory of Benjamin Neel (New York University, New York, New York, USA).

Tumor implantation, treatment, and harvest. Luciferase-tagged B16F10 melanoma cells $\left(1 \times 10^{5}\right)$ were implanted subcutaneously in 8 -week-old mice (randomized, both male and female). Subsequent tumors were harvested $1,3,7,10$, or 14 days after implantation following cardiac perfusion with PBS and then 4\% PFA. For survival studies, tumors were harvested when they reached $1.5 \mathrm{~cm}$ in any direction. Where indicated, mice received intraperitoneal injection every other day with $100 \mu \mathrm{l}$ of $0.4 \mathrm{mg} / \mathrm{ml}$ resiquimod (ALZ-420-038-M005, Enzo) or vehicle (PBS, Gibco). Resiquimod $(0.4 \mathrm{mg} / \mathrm{ml})$ was prepared by reconstituting $5 \mathrm{mg}$ resiquimod with $1 \mathrm{ml}$ DMSO (Sigma-Aldrich), followed by addition of $11.5 \mathrm{ml}$ PBS (Gibco).

Immunofluorescence staining, confocal imaging, and quantitation. Immunofluorescence staining on frozen sections $(7 \mu \mathrm{m})$ was performed using primary antibodies to Pros1 (sc-27027, Santa Cruz Biotechnology Inc.), Gas6 (sc-16660, Santa Cruz Biotechnology Inc.), luciferase (sc-32896, Santa Cruz Biotechnology Inc.), IL1 (AF-400-NA, R\&D Systems), IL6 (AF-406-NA, R\&D Systems), and iNOS (sc-49055, Santa Cruz Biotechnology Inc.); and Alexa Fluor 488- or Alexa Fluor 594-labeled secondary antibodies (Molecular Probes). Labeled sections were imaged using an LSM 710 Spectral Confocal Laser Scanning Microscope (Zeiss). Five independent images for each tumor were obtained, and colocalization analysis of confocal images was performed using Image J software (NIH).
Immunohistochemistry and quantitation. Immunohistochemistry was performed on formalin-fixed, paraffin-embedded sections $(4 \mu \mathrm{m})$ using primary antibodies to F4/80 (MCA497RT, AbD Serotec), iNOS (ABN26, Sigma-Aldrich), arginase (sc-20150, Santa Cruz Biotechnology Inc.), CD11b (ab133357, Abcam), CD4 (14-9766, eBioscience), CD8a (14-0808, eBioscience), FoxP3 (14-5773, eBioscience), or cleaved caspase-3 (9664, Cell Signaling Technology) on a Discovery Ultra Automated IHC staining system (Ventana). Sections were imaged on a ScanScope XT (Aperio) and quantitated using Aperio software.

Peritoneal macrophage isolation, culture, and treatment. Peritoneal or thioglycollate-induced macrophage isolation was conducted as described previously (54) with the following modifications. Harvest was performed on 8-week-old mice (randomized, both male and female; 000664, The Jackson Laboratory). For peritoneal macrophages, PBS (Gibco) was injected into the peritoneal cavity. After removal, cells were pelleted; resuspended in DMEM-H, 10\% FBS, 1× penicillin/streptomycin (Gibco); and plated. After 1 hour, cells were washed once with PBS and cultured in DMEM/F12, 10\% FBS, 1× penicillin/streptomycin, $20 \mathrm{mg} / \mathrm{ml} \mathrm{M-CSF}$ (Prospec) for 3 days prior to treatment. Macrophages were treated for 24 hours with $100 \mathrm{ng} / \mathrm{ml}$ IFN- $\gamma$ (BioLegend), $50 \mathrm{ng} / \mathrm{ml}$ LPS (Sigma-Aldrich), $1 \mu \mathrm{g} / \mathrm{ml}$ human recombinant Pros1 (Sino Biologicals), $10 \mathrm{nM}$ colivelin (Santa Cruz Biotechnology Inc.), $300 \mathrm{ng} / \mathrm{ml}$ anisomycin (Santa Cruz Biotechnology Inc.), $5 \mu \mathrm{M}$ BVT948 (Sigma-Aldrich), $200 \mu \mathrm{M}$ PTP inhibitor III (Santa Cruz Biotechnology Inc.), $5 \mu \mathrm{M}$ NSC87877 (Santa Cruz Biotechnology Inc.), or $125 \mathrm{ng} / \mathrm{ml}$ resiquimod (ALZ-420-038-M005, Enzo), as described in the text. All additions to the cultured cells were made at the same time, and there was no TAM ligand pretreatment as has been described elsewhere (55). Transwell assays were conducted in Costar $0.4 \mu \mathrm{M}$ polyester membrane insert culture plates (Thermo Fisher Scientific).

Cell culture. B16F10 cells (UNC Tissue Culture Facility) were cultured in DMEM-H, 10\% FBS, $1 \times$ penicillin/streptomycin. In Transwell assays, B16F10 cells were plated in DMEM/F12,10\% FBS, $1 \times$ penicillin/ streptomycin, $20 \mathrm{mg} / \mathrm{ml} \mathrm{M-CSF}$ for culture with murine macrophages. Lewis lung carcinoma (CRL-1642, ATCC), PyMT (CRL-3278, ATCC), KPC2713 (Yuliya Pylayeva-Gupta laboratory, UNC), KPC4662 (Yuliya Pylayeva-Gupta laboratory), KPPC4548 (Yuliya Pylayeva-Gupta laboratory), G361 (Douglas Graham laboratory, Emory University, Atlanta, Georgia, USA), HMCB (Douglas Graham laboratory), MCF7 (Shumang Feng, UNC), MDA-MB-231 (Shumang Feng, UNC), LNCap (Ling Cai, UNC), and PC3 (Ling Cai) were cultured in either DMEM-H or RPMI supplemented with $10 \%$ FBS and 1× penicillin/streptomycin. SC cells (CRL-9855, ATCC) were cultured in IMDM, 10\% FBS, 1× penicillin/ streptomycin. In Transwell assays with SC cells, tumor cell lines were plated in IMDM, 10\% FBS, 1× penicillin/streptomycin.

CRISPR. Target site guides were designed using the CRISPR Design web site (Feng Zhang laboratory, MIT, Cambridge, Massachusetts, USA; http://crispr.mit.edu). Guides were cloned into the U6 expression plasmid (51133, Addgene) and cotransfected with the Cas9 plasmid (41815, Addgene), followed by selection with $2 \mu \mathrm{g} / \mathrm{ml}$ puromycin for 6 days. Single cells were isolated by limiting dilution and screened for genetic and functional deletion of Pros1.

qRT-PCR. RNA was isolated using the SV Total RNA Isolation System (Promega) and cDNA prepared using the Reverse Transcription System (Promega) with a 1-hour reverse transcription step. qRTPCR was performed using the SYBR and Fluorescein Kit (Bioline) on 
a QuantStudio 7 Flex Real-Time PCR System (Thermo Fisher Scientific) using the following thermal cycling profile: $95^{\circ} \mathrm{C}, 10$ minutes; 40 cycles $-95^{\circ} \mathrm{C}, 15$ seconds, $60^{\circ} \mathrm{C}, 1$ minute. Analysis was conducted using the $\Delta \Delta \mathrm{CT}$ method. As we are not aware of previously reported detection of Tyro3 in peritoneal macrophages, the primer sequences (5' to $3^{\prime}$ ) used to detect Tyro3 and GAPDH were as follows: Tyro3 F, GTGAAGgAtgGgGAgGAAAC, Tyro3 R, GTGgCACCGCCAGATCTTTT; GAPDH F, TCGGTGTGAACGGATTTGGC, GAPDH R, GTGCCGTTGAATTTGCCGTG.

ELISA. Pros1 ELISA (MBS906634, MyBioSource) was conducted per the manufacturer's instructions.

Coimmunoprecipitation and Western blot analysis. Coimmunoprecipitation was carried out using antibodies for c-Jun (9165S, Cell Signaling Technology), Mer (4319S, Cell Signaling Technology), or PTP1b (sc-14021, Santa Cruz Biotechnology Inc.). Isolated protein was run on 10\% Mini-PROTEAN TGX Stain-Free gels (Bio-Rad) in the MiniPROTEAN Tetra System (Bio-Rad) and transferred to a 0.2- $\mu \mathrm{m}$ nitrocellulose Trans-Blot Turbo Transfer Pack (Bio-Rad) using a Trans-Blot Turbo device (Bio-Rad) and probed with antibodies for p38 $\alpha$ (sc-535, Santa Cruz Biotechnology Inc.), p38 $\gamma$ (sc-366013, Santa Cruz Biotechnology Inc.), p-p38 (9211S, Cell Signaling Technology), p-Stat3 (9134S, Cell Signaling Technology), Stat3 (sc-482, Santa Cruz Biotechnology Inc.), Mer (sc-67280, Santa Cruz Biotechnology Inc.), PTP1b (sc14021, Santa Cruz Biotechnology Inc.), p-AKT (9018S, Cell Signaling Technology), AKT (4691S, Cell Signaling Technology), p-MEK (9121S, Cell Signaling Technology), MEK (9146S, Cell Signaling Technology), p-Stat1 (9167S, Cell Signaling Technology), Stat1 (9172, Cell Signaling Technology), p-Stat6 (9361S, Cell Signaling Technology), Stat6 (9362, Cell Signaling Technology), p-ERK (9101S, Cell Signaling Technology), ERK (9102S, Cell Signaling Technology), and $\beta$-tubulin (2128, Cell Signaling Technology). Detection was performed using ECL HRP-linked anti-rabbit IgG (GE Healthcare) and WesternBright ECL (Advansta). Blots were imaged on a ChemiDoc MP Imaging System (Bio-Rad) and quantitated with ImageJ software (NIH).

ChIP. ChIP was performed using a ChIP Assay Kit (17-295, Millipore) per the manufacturer's instructions using c-Jun antibody (9165S, Cell Signaling Technology) and validated primer sets [GPM1049162(-) 01A (IL1) and GPM1036981(-)01A (IL6), QIAGEN].

Cell proliferation assay. Cell numbers were measured over time using the CellTiter 96 AQueous Assay (G5430, Promega). $2 \times 10^{3}$ cells were added to wells of a 96 -well plate. Cells were incubated 24 and 48 hours at $37^{\circ} \mathrm{C}$, then $20 \mu \mathrm{l} /$ well of MTS solution was added. After 1 hour at $37^{\circ} \mathrm{C}$, absorbance at $490 \mathrm{~nm}$ was measured using a plate reader.

Flow cytometry. Cultured macrophages were released into suspension by incubation with Versene, washed, and resuspended in flow buffer (0.5\% BSA, 2 mM EDTA in PBS, pH 7.2). Macrophages were stained using Axl-PE (FAB8541P, R\&D Systems), Mer-PE (151506, BioLegend), or Tyro3-PE (FAB759P, R\&D Systems) for 30 minutes on ice, washed, and resuspended in flow buffer for analysis with an LSRFortessa (BD Biosciences). Data were analyzed using FlowJo software.

Pros1 expression and mutations in human tumors. Analysis of human tumor data was conducted using the cBioPortal for Cancer Genomics web site (http://www.cbioportal.org).

Statistics. Statistical analysis was performed using GraphPad software (Prism) and Student's $t$ test (2-tailed). A P value less than 0.05 was considered statistically significant. Graphs present the mean value \pm SEM. The number of independent replicate studies performed to arrive at the total sample size $(n)$ is described in respective figure legends. Where not explicitly stated, the results are from one study with the indicated sample size.

Study approval. All animal studies were approved by the UNC Institutional Animal Care and Use Committee.

\section{Author contributions}

EU conceptualized, planned, and performed experiments; analyzed data; and prepared the manuscript. LC designed and constructed the CRISPR-modified cell line. AH conducted flow cytometry analysis. DH performed cell proliferation assays. CS assisted EU in various aspects of the project. HSE helped conceptualize the study and prepared the manuscript.

\section{Acknowledgments}

We thank Charlene Santos and the UNC Animal Studies Core for assistance with animal studies. We also thank UNC Lineberger core facilities, including the Animal Histopathology Core, Translational Pathology Laboratory, Histology Core, Microscopy Service Laboratory, and Flow Cytometry Core. We gratefully acknowledge Benjamin Neel for providing PTP1b-KO mice for the Transwell coculture assays. We also thank Douglas Graham, Yuliya PylayevaGupta, Shumang Feng, and Ling Cai for donations of murine and human tumor cell lines. EU was supported by a UNC Lineberger Integrated Training in Cancer Model Systems (ITCMS) Training Grant (T32 CA009156) and an American Cancer Society Postdoctoral Fellowship (128770-PF-15-216-01-LIB). Studies were supported by grants to HSE from a Saban Team Science Award from the Melanoma Research Alliance, Public Health Service (PHS) grant CA205398, and the Breast Cancer Research Foundation.

Address correspondence to: H. Shelton Earp, UNC Lineberger Comprehensive Cancer Center, 450 West Drive, Chapel Hill, North Carolina 27599, USA. Phone: 919.966.1185; Email: shelton_earp@med.unc.edu.
1. Pardoll DM. The blockade of immune checkpoints in cancer immunotherapy. Nat Rev Cancer. 2012;12(4):252-264.

2. Zou W. Immunosuppressive networks in the tumour environment and their therapeutic relevance. Nat Rev Cancer. 2005;5(4):263-274.

3. Coussens LM, Zitvogel L, Palucka AK. Neutralizing tumor-promoting chronic inflammation: a magic bullet? Science. 2013;339(6117):286-291.

4. Murray PJ, Wynn TA. Protective and pathogenic functions of macrophage subsets. Nat Rev Immu- nol. 2011;11(11):723-737.

5. Mantovani A, Sica A, Sozzani S, Allavena P, Vecchi A, Locati M. The chemokine system in diverse forms of macrophage activation and polarization. Trends Immunol. 2004;25(12):677-686.

6. Pollard JW. Tumour-educated macrophages promote tumour progression and metastasis. Nat Rev Cancer. 2004;4(1):71-78.

7. Joyce JA, Pollard JW. Microenvironmental regulation of metastasis. Nat Rev Cancer. 2009;9(4):239-252.
8. Camenisch TD, Koller BH, Earp HS, Matsushima GK. A novel receptor tyrosine kinase, Mer, inhibits TNF-alpha production and lipopolysaccharide-induced endotoxic shock. J Immunol. 1999;162(6):3498-3503.

9. Scott RS, et al. Phagocytosis and clearance of apoptotic cells is mediated by MER. Nature. 2001;411(6834):207-211.

10. Rothlin CV, Ghosh S, Zuniga EI, Oldstone MB, Lemke G. TAM receptors are pleiotropic inhibitors of the innate immune response. Cell. 
2007;131(6):1124-1136.

11. Lemke G, Rothlin CV. Immunobiology of the TAM receptors. Nat Rev Immunol. 2008;8(5):327-336.

12. Tsou WI, et al. Receptor tyrosine kinases, TYRO3, AXL, and MER, demonstrate distinct patterns and complex regulation of ligand-induced activation. J Biol Chem. 2014;289(37):25750-25763.

13. Cook RS, et al. MerTK inhibition in tumor leukocytes decreases tumor growth and metastasis. JClin Invest. 2013;123(8):3231-3242.

14. Stanford JC, et al. Efferocytosis produces a prometastatic landscape during postpartum mammary gland involution. JClin Invest. 2014;124(11):4737-4752.

15. Zizzo G, Hilliard BA, Monestier M, Cohen PL. Efficient clearance of early apoptotic cells by human macrophages requires M2c polarization and MerTK induction. JImmunol. 2012;189(7):3508-3520.

16. Iwai Y, Ishida M, Tanaka Y, Okazaki T, Honjo T, Minato N. Involvement of PD-L1 on tumor cells in the escape from host immune system and tumor immunotherapy by PD-L1 blockade. Proc Natl Acad Sci U S A. 2002;99(19):12293-12297.

17. Cheng X, Zhao Z, Ventura E, Gran B, Shindler KS, Rostami A. The PD-1/PD-L pathway is upregulated during IL-12-induced suppression of EAE mediated by IFN-gamma. J Neuroimmunol. 2007;185(1-2):75-86.

18. Mühlbauer M, et al. PD-L1 is induced in hepatocytes by viral infection and by interferon-alpha and -gamma and mediates $\mathrm{T}$ cell apoptosis. JHepatol. 2006;45(4):520-528.

19. Li Y, et al. Axl as a potential therapeutic target in cancer: role of Axl in tumor growth, metastasis and angiogenesis. Oncogene. 2009;28(39):3442-3455.

20. Zagórska A, Través PG, Lew ED, Dransfield I, Lemke G. Diversification of TAM receptor tyrosine kinase function. Nat Immunol. 2014;15(10):920-928.

21. Lu Q, et al. Tyro-3 family receptors are essential regulators of mammalian spermatogenesis. Nature. 1999;398(6729):723-728.

22. Seitz HM, Camenisch TD, Lemke G, Earp HS, Matsushima GK. Macrophages and dendritic cells use different Axl/Mertk/Tyro3 receptors in clearance of apoptotic cells. JImmunol. 2007;178(9):5635-5642.

23. Graham DK, DeRyckere D, Davies KD, Earp HS. The TAM family: phosphatidylserine sensing receptor tyrosine kinases gone awry in cancer. Nat Rev Cancer. 2014;14(12):769-785.

24. Kang YJ, et al. Macrophage deletion of p38alpha partially impairs lipopolysaccharide-induced cellular activation. J Immunol. 2008;180(7):5075-5082.

25. Zarubin T, Han J. Activation and signaling of the p38 MAP kinase pathway. Cell Res. 2005;15(1):11-18.

26. Rothlin CV, Ghosh S, Zuniga EI, Oldstone MB, Lemke G. TAM receptors are pleiotropic inhibitors of the innate immune response. Cell. 2007;131(6):1124-1136.

27. Keating MJ, et al. Fludarabine: a new agent with major activity against chronic lymphocytic leukemia. Blood.1989;74(1):19-25.

28. Ma Q, Guin S, Padhye SS, Zhou YQ, Zhang RW, Wang MH. Ribosomal protein S6 kinase (RSK)-2 as a central effector molecule in $\mathrm{RON}$ receptor tyrosine kinase mediated epithelial to mesenchymal transition induced by macrophage-stimulating protein. Mol Cancer. 2011;10:66.

29. Müller J, Sperl B, Reindl W, Kiessling A, Berg T. Discovery of chromone-based inhibitors of the transcription factor STAT5. Chembiochem. 2008;9(5):723-727.

30. Moisan A, et al. White-to-brown metabolic conversion of human adipocytes by JAK inhibition. Nat Cell Biol. 2015;17(1):57-67.

31. Kang YJ, et al. Macrophage deletion of p38alpha partially impairs lipopolysaccharide-induced cellular activation. JImmunol. 2008;180(7):5075-5082.

32. Pramanik R, et al.p38 isoforms have opposite effects on AP-1-dependent transcription through regulation of c-Jun. The determinant roles of the isoforms in the p38 MAPK signal specificity. JBiol Chem. 2003;278(7):4831-4839.

33. Zhao TC, Zhang L, Liu JT, Guo TL. Disruption of Nox 2 and TNFRp55/p75 eliminates cardioprotection induced by anisomycin. Am JPhysiol Heart Circ Physiol. 2012;303(10):H1263-H1272.

34. Chiba T, et al. Development of a femtomolaracting humanin derivative named colivelin by attaching activity-dependent neurotrophic factor to its $\mathrm{N}$ terminus: characterization of colivelinmediated neuroprotection against Alzheimer's disease-relevant insults in vitro and in vivo JNeurosci. 2005;25(44):10252-10261.

35. Través PG, et al. Pivotal role of protein tyrosine phosphatase 1B (PTP1B) in the macrophage response to pro-inflammatory and anti-inflammatory challenge. Cell Death Dis. 2014;5:e1125.

36. Xu H, et al. Phosphatase PTP1B negatively regulates MyD88- and TRIF-dependent proinflammatory cytokine and type I interferon production in TLR-triggered macrophages. Mol Immunol. 2008;45(13):3545-3552.

37. Medgyesi D, et al. The protein tyrosine phosphatase PTP1B is a negative regulator of CD40 and BAFF-R signaling and controls B cell autoimmunity. JExp Med. 2014;211(3):427-440.

38. Carbone $\mathrm{CJ}$, et al. Protein tyrosine phosphatase $1 \mathrm{~B}$ is a key regulator of IFNAR1 endocytosis and a target for antiviral therapies. Proc Natl Acad Sci U S A. 2012;109(47):19226-19231.

39. Bentires-Alj M, Neel BG. Protein-tyrosine phosphatase $1 \mathrm{~B}$ is required for HER2/Neu-induced breast cancer. Cancer Res. 2007;67(6):2420-2424

40. Julien SG, et al. Protein tyrosine phosphatase 1B deficiency or inhibition delays ErbB2-induced mammary tumorigenesis and protects from lung metastasis. Nat Genet. 2007;39(3):338-346.
41. Liljebris $\mathrm{C}$, et al. Oxidation of protein tyrosine phosphatases as a pharmaceutical mechanism of action: a study using 4-hydroxy-3,3-dimethyl2H-benzo[g]indole-2,5(3H)-dione. JPharmacol Exp Ther. 2004;309(2):711-719.

42. Kanda M, et al. Glutaredoxin modulates plateletderived growth factor-dependent cell signaling by regulating the redox status of low molecular weight protein-tyrosine phosphatase. J Biol Chem. 2006;281(39):28518-28528.

43. Chen L, et al. Discovery of a novel shp2 protein tyrosine phosphatase inhibitor. Mol Pharmacol. 2006;70(2):562-570.

44. Clausen BE, Burkhardt C, Reith W, Renkawitz $\mathrm{R}$, Förster I. Conditional gene targeting in macrophages and granulocytes using LysMcre mice. Transgenic Res. 1999;8(4):265-277.

45. Madisen L, et al. A robust and high-throughput Cre reporting and characterization system for the whole mouse brain. Nat Neurosci. 2010;13(1):133-140.

46. Cabezón R, et al. MERTK as negative regulator of human T cell activation. J Leukoc Biol. 2015;97(4):751-760.

47. Hemmi H, et al. Small anti-viral compounds activate immune cells via the TLR7 MyD88dependent signaling pathway. Nat Immunol. 2002;3(2):196-200.

48. Zaidi MR, Merlino G. The two faces of interferon- $\gamma$ in cancer. Clin Cancer Res. 2011;17(19):6118-6124

49. Carrera Silva EA, et al. T cell-derived protein $S$ engages TAM receptor signaling in dendritic cells to control the magnitude of the immune response. Immunity. 2013;39(1):160-170.

50. Vallabhapurapu SD, et al. Variation in human cancer cell external phosphatidylserine is regulated by flippase activity and intracellular calcium. Oncotarget. 2015;6(33):34375-34388.

51. Goldstein BJ, Bittner-Kowalczyk A, White MF, Harbeck M. Tyrosine dephosphorylation and deactivation of insulin receptor substrate-1 by protein-tyrosine phosphatase 1B. Possible facilitation by the formation of a ternary complex with the Grb2 adaptor protein. J Biol Chem. 2000;275(6):4283-4289.

52. Zhang S, Zhang ZY. PTP1B as a drug target: recent developments in PTP1B inhibitor discovery. Drug Discov Today. 2007;12(9-10):373-381.

53. Mazzoni A, et al. Myeloid suppressor lines inhibit $\mathrm{T}$ cell responses by an NO-dependent mechanism. J Immunol. 2002;168(2):689-695.

54. Ray A, Dittel BN. Isolation of mouse peritoneal cavity cells. J Vis Exp. 2010;(35):1488.

55. Tibrewal N, et al. Autophosphorylation docking site Tyr-867 in Mer receptor tyrosine kinase allows for dissociation of multiple signaling pathways for phagocytosis of apoptotic cells and down-modulation of lipopolysaccharideinducible NF-kappaB transcriptional activation. J Biol Chem. 2008;283(6):3618-3627. 